\title{
Anesthetic considerations of Conn syndrome: A case presentation and mini-review the anesthesiologist and Conn syndrome
}

\author{
Arber Jano $^{1}$, Rudin Domi ${ }^{1}$, Leart Berdica ${ }^{2}$, Hektor Sula ${ }^{1}$, Ilir Ohri ${ }^{1}$ \\ ${ }^{1}$ Department of Anesthesia and Intensive Care, "Mother Teresa" University Hospital Center, Tirana, Albania \\ ${ }^{2}$ Department of Morphopathology, "Mother Teresa" University Hospital Center, Tirana, Albania \\ Email address: \\ arber_jano@yahoo.com (A. Jano), rdomi73@yahoo.it (R. Domi), leartberdica@ymail.com (L. Berdica), hektorsula@yahoo.com (H. Sula)
}

\section{To cite this article:}

Arber Jano, Rudin Domi, Leart Berdica, Hektor Sula, Ilir Ohri. Anesthetic Considerations of Conn Syndrome: A Case Presentation and Mini-Review the Anesthesiologist and Conn Syndrome. Clinical Medicine Research. Vol. 3, No. 5, 2014, pp. 132-135.

doi: 10.11648/j.cmr.20140305.14

\begin{abstract}
Conn syndrome is characterized by increased secretion of aldosterone. Hyperaldosteronism induces sodium and water retention, hypertension, decreased potassium blood level, muscle weakness, and fatigue. The diagnosis is usually confirmed by decreased renin level, increased aldosteron level, hypokalemia, and imagining tools. Perioperative care consists on blood pressure and hypervolemia control, spironolactone administration, and preoperative potassium correction. We present a case scheduled for left adrenalectomy for Conn syndrome, 5 years after the right adrenalectomy was performed. The surgery and postoperative period were uneventful and the patient was discharged in 6-th postoperative day referring to the endocrinologist. A good cooperation between the anesthesiologist, endocrinologist, and surgeon is strongly recommended.
\end{abstract}

Keywords: Adrenal Gland, General Anesthesia, Hypokalemia, Conn Syndrome

\section{Introduction}

Hyperaldosteronism is characterized by an excess of aldosterone. Hyperaldosteronism can be divided in primary and secondary one. The secondary hyperaldosteronism may be due to severe liver diseases, nephritic syndrome, and cardiac failure. The primary form (Conn syndrome) presents the excess of aldosterone due to an adrenal gland disease. In the majority of cases $(60 \%)$ a unilateral adenoma may be verified, whereas bilateral adrenal hyperplasia is faced in $30 \%$ of cases. Patients with Conn syndrome may have decreased level of renin and increased level of aldosterone. It is more common in females than males $(1,2)$, and is the cause of $5-13 \%$ of secondary hypertension and less that $1 \%$ of essential one (2).

Hypertension, muscle cramps, fatigue, decreased blood potassium levels, increased sodium, and metabolic alkalosis are the main clinical features. Computerized tomography (CT) examination, ultrasound, magnetic resonance imagining (MRI), decreased renin, and increased aldosterone can afford the diagnosis. Spironolactone and potassium containing fluids can be used, but surgical treatment seems to be the definitive approach.

\section{Case Presentation}

V.S. 40 year old Albanian women (body weight $76 \mathrm{~kg}$, height $171 \mathrm{~cm}$, body mass index 26), was consulted by an endocrinologist because of hypertension (170-240/100-115 $\mathrm{mmHg}$, muscle weakness, fatigue, hypokalemia, and hypermatremia. The patient had right adrenalectomy 5 years ago for pheochromocytoma. Conn syndrome of left adrenal gland was suspected. She undergone to an angio-CT, ultrasound examination, during which was verified a left adrenal hyperplasia (Figure 1, 2). The blood -potassium level was $2.28 \mathrm{mmol} / 1$ (normal values 3.5-5.1 mmol. $\mathrm{I}^{-1}$ ), sodium 156 mmol. $\mathrm{I}^{-1}$ (normal values 136-148 $\mathrm{mmol}^{-1} \mathrm{l}^{-1}$ ), aldosterone 192.71 pg. $\mathrm{ml}^{-1}$ (normal values $20-180$ pg.ml ${ }^{-1}$ ), and cortisol 150.049 ng. $\mathrm{ml}^{-1}$ (normal values $55-288$ ng. ml ${ }^{-1}$ ). The clinical assessment, chest $\mathrm{x}$-ray and other biochemistry findings were normal. Preoperative hypertension was treated with metoprolol, and alphametildopa. Spironolactone (100 mg twice daily) was preoperatively administered. The potassium containing fluids were preoperatively administered in order to 
correct hypokalemia. The patient was premedicated with oral Diazepam $10 \mathrm{mg}$ the night before the surgery and morphine sulfate $10 \mathrm{mg}$ intramuscularly 30 minutes before the induction of anesthesia, continuing of course all the preoperative treatment including spironolactone as well. Preoperative period was uneventful. In operating room after peripheral venous cannula was inserted, right radial artery and right internal jugular vein were cannulated with $20 \mathrm{G}$ venous catheter $7.5 \mathrm{~F}$ central venous catheter. The anesthesia's induction was made with (Fentanyl $4 \mathrm{mcg} \cdot \mathrm{kg}^{-1}$, Propofol 2 mg. $\mathrm{kg}^{-1}$, cis-atracurium $\left.0.15 \mathrm{mg} . \mathrm{kg}^{-1}\right)$. Monitoring included invasive arterial monitoring, central venous pressure, electrocardiogram, temperature, urine output, end-Tidal carbon dioxide, and intraoperatively electrolytes. The anesthesia was maintained with sevoflurane, fentanyl and cis-atracurium as needed. An open radical left adrenalectomy (Figure 3) was performed, and after the adrenal gland vein was clamped 100 mg endovenous hydrocortisone was administered. Postoperatively spironolactone was discontinued and $100 \mathrm{mg}$ of hydrocortisone succinate every 8 hours was administered. The hypertension also was stabilized and only a small dose of metoprolol was necessary. The cortisol and potassium levels were $61 \mathrm{ng} . \mathrm{ml}^{-1}$ and $4.3 \mathrm{mmol}^{-1} \mathrm{l}^{-1}$ respectively. The pathology examination showed the interface between the cortico-adrenal adenoma secreting aldosterone (Figure 4- 8). The pattern of the tumor is of nest of pale cells packed closely to each other resembling the glomerulosa layer of the adrenal gland. The surgery and postoperative period were uneventful and the patient was discharged in 6-th postoperative day referring to the endocrinologist.
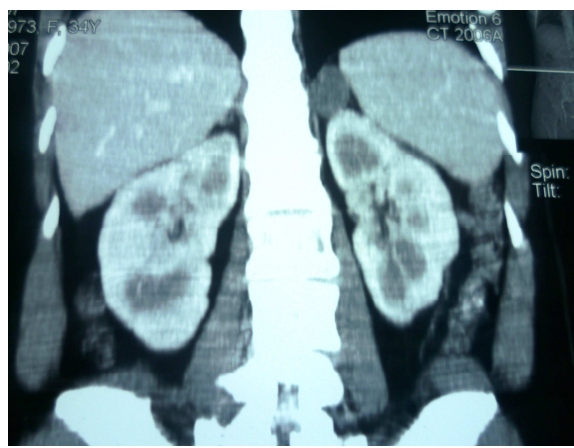

Figure 1. Angio-CT examination

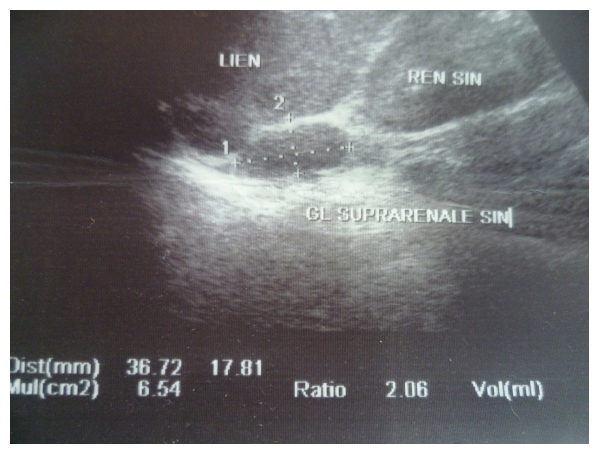

Figure 2. The ultrasound examination showed the hypertrophied left adrenal gland

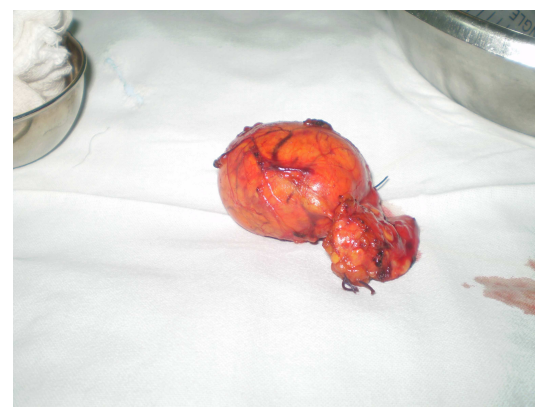

Figure 3. The removed left adrenal gland
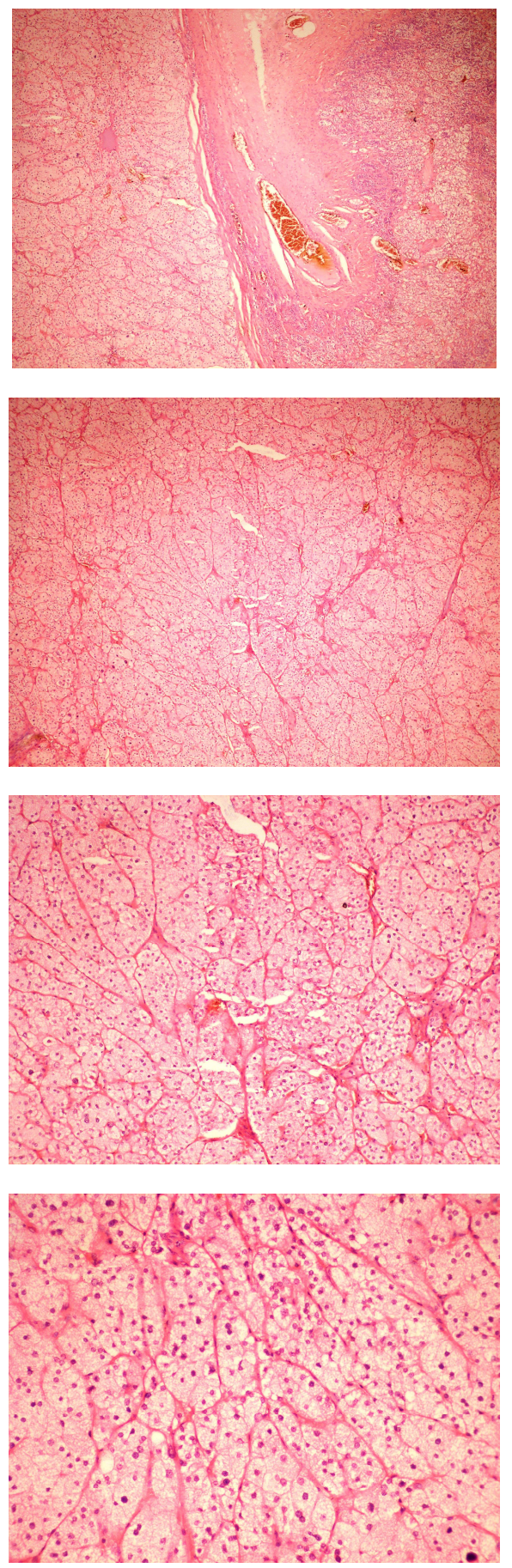


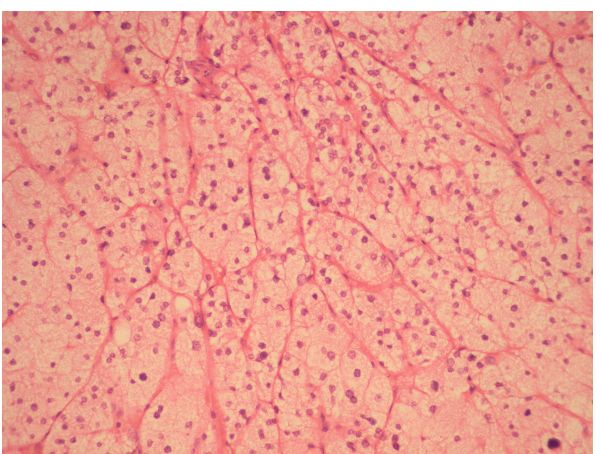

Figure 4-8. The pathology examination of the removal adrenal tumor

\section{Discussion}

As afore mentioned hyperaldosteronism can be two forms: primary and secondary hyperaldosteronism. Primary hyperaldosteronism is known as Conn syndrome resulting from unilateral or bilateral adrenal gland tumors (3-5).

Secondary hyperaldosteronism is due to increased levels of renin, inducing the renin-aldosterone axis activitation. These pathophysiologic changes are usually present in severe cardiac failure, nephritic syndrome, and advanced liver disease. Our patient was diagnosed with Conn syndrome as a result of unilateral adrenal gland adenoma, clinical features, and pathology examination.

The clinical features include systemic hypertension, metabolic alkalosis, hypokalemia, increased urinary excretion of potassium, hypernatremia, fatigue, muscle cramps, and skeletal muscle weakness. Systemic hypertension (often increased diastolic pressure) can be result of aldosterone- induced sodium and water retention. This hypertension is often resistant of pharmacologic treatment. Fatigue, muscle cramps, and muscle weakness are presented due to hypokalemia. Our patient had all the clinical features (hypertension, hypokalemia, increased blood level of aldosterone), strongly suggested the endocrinology abnormality.

Diagnosing Conn syndrome is quite simple. Clinical features can suggest the diagnosis but are not specific, but Conn syndrome is suspected in presence of diastolic hypertension, low plasma renin and high plasma aldosterone not able to be suppressed by fluid challenge (6). After this scenario the imagining methods and biochemistry examination are of great importance. Biochemistry examinations include renin and aldosterone blood level, potassium and sodium plasma concentration. Increased renin level can be faced in secondary hyperaldosteronism, whereas decreased renin is usually found in Conn syndrome, associated with high aldosterone level. Aldosterone increases the effect of catecholamines, due to noradrenaline re-uptake blocking effect, and it predisposes to myocardial fibrosis resulting in arrhythmias and myocardial ischaemia (7-9). Hypokalemia is a constant finding, whereas hypernatremia can commonly be faced. Our patient had hypertension, fatigue, and muscle weakness, associated with increased aldosterone level and hypokalemia. Imagining examinations include ultrasound, angio CT scan, and MRI. Our patient was first undergone to ultrasound examination, followed by angio CT scan and MRI. The diagnosis was confirmed by clinical features, biochemistry findings, and supported by imagining examinations.

The anesthesiologist must deal with intraoperative hemodynamic changes and hypokalemia. It is well-known that hypokalemia and metabolic alkalosis may prolong the action of neuromuscular blocking agents, inducing bradycardia. Hypokalemia may be worsened by respiratory alkalosis (hyperventilation) and by sevoflurane induced polyuria. The anesthesiologist must avoid hyperventilation and sevoflurane use as an inhalation anesthetic drug. The manipulation of adrenal gland during dissection and resection may lead to catecholamine release from the adrenal medulla with resultant hemodynamic fluctuations $(9,10)$. It is recently reported that Conn syndrome can produce brisk and untreatable intraoperative hypertension (11). The preoperative fluid volume status evaluation (the presence of orthostatism, increased heart rate, blood pressure, increased hematocrite etc) can detect hypovolemia. Hypovolemia is a rare condition and can be multifactorial including diuretics' use, anesthetic drugs' effects, positive pressure ventilation, laparoscopic approach, and the patient's position. Both hypervolemia and hypovolemia must be aggressively treated.

Laparoscopic approach remains gold standard $(12,13)$. It is generally accepted that laparoscopic approach may decrease postoperative cardiac and respiratory complications, less postoperative pain, and early ambulation (14).

Supplementation of hydrocortisone was another issue of our case, that anesthesiologist must deal with. Cortisol administration is helpful in perioperative hypoadrenocorticism or in chronic steroid administration (15). Adrenal suppression induces hypotension, decreased cardiac output, hyponatremia, and hypoglycemia. It is mandatory to control cortisol level in preoperatively inadequate cotisol secretion patient, and cortisol supplementation as well. Etomidate must be avoided because it interferes with cortisol synthesis (16). Our patient remained without adrenal gland so we supplemented hydrocortisone as the adrenal gland vein was clamped.

As a conclusion, Conn syndrome presents different problems to the anesthesiologist. The anesthesiologist must deal with hypertension, hypervolemia, hypokalemia, and depending case by case with cortisol supplementation. A good cooperation between the anesthesiologist, endocrinologist, and surgeon is strongly recommended.

\section{List of Abbreviations}

Computerized tomography (CT), magnetic resonance imagining (MRI) 


\section{Compenting Interest}

The authors declare that they have no competing interests

\section{References}

[1] Williams, Dluhy RG. Disorders of the Adrenal Cortex. In: Fauci A, Braunwald E, Kasper D, Hauser S, Longo D, Jameson J, Loscalzo J, ed. Harrison's Principles of Internal Medicine. 17th Ed Chapter. McGraw Hill Professional Publishing, 2008:2259-60.

[2] Michael FR, Fleisher LA. Anesthetic Implications of Concurrent Diseases. In: In: Miller RD, ed. Miller's Anesthesia. 7th ed. Philadelphia: Churchill Livingstone, 2009: $1067-1150$

[3] Calhoun DA: Aldosteronism and hypertension. Clin J Am Soc Nephrol 2006;1:1039-1045,

[4] Wheeler MH, Harris DA. Diagnosis and management of primary aldosteronism. World J Surg.2003; 27(6):627-31

[5] Mattsson C, Young WF Jr. Primary aldosteronism: diagnostic and treatment strategies. Nat Clin Pract Nephrol. 2006; 2(4):198-208

[6] Quinkler M, Reincke M. Modern pharmacological aspects of hyperaldosteronism therapy. Internist (Berlin). 2006; 47(9):953-959

[7] Funder JW. Aldosterone, salt and cardiac fibrosis. Clinical and Experimental Hypertension 1997; 19: 885-99
[8] Zannad F. Aldosterone and heart failure. European Heart Journal 1995; 16: 98-102.

[9] Winship SM, Winstanley JHR, Hunter JM. Anaesthesia for Conn's syndrome. Anaesthesia, 1999; 54:569-573

[10] Domi R, Sula H. Pheochromocytoma, the challenge to anesthesiologist. J Endocrinol Metab 2011; 1(3):97-100

[11] Gockel I, Heintz A, Kentner R, Wetner C, Junginger Th: Changing pattern of the intraoperative blood pressure during endoscopic adrenalectomy in patients with Conn's syndrome. Surg Endosc. 2005; 19(11):1491-7

[12] Lertakyamanee N, Somprakit P, Buranakijaroen P. Anesthesia and laparoscopic adrenalectomy for primary aldosteronism. J Med Assoc Thai. 2001; 84(6):798-803

[13] Edwin B, Raeder I, Trondsen E, Kaaresen R, Buanes T: Outpatient Laparoscopic Adrenalectomy in patients with Conn's Syndrome. Surg Endosc. 2001; 15 (6):589-91

[14] Domi R. Cushing' surgery: Role of the anesthesiologist. Indian J Endocr Metab 2011; 15:322-8

[15] Shaikh S, Verna H, Yadav N, Jauhari M, Bullangowda J. Application of steroid in clinical practice: a review. ISRN Anesthesiology, Volume 2012, Art ID 985495

[16] Absolon A, Pledger D, Kong A. Adrenocortical function in critical ill patient $24 \mathrm{~h}$ after a single dose or etomidate. Anaesthesia 1999; 54:861-7 